\title{
Method to Reduce the False-Positive Rate of Loss of Resistance in the Cervical Epidural Region
}

\author{
Young Uk Kim,, ${ }^{1}$ Doohwan Kim, ${ }^{2}$ Jun Young Park, ${ }^{2}$ Jae-Hyung Choi, ${ }^{2}$ \\ Ji Hyun Kim, ${ }^{2}$ Heon-Yong Bae, ${ }^{2}$ Eun-Young Joo, ${ }^{2}$ and Jeong Hun Suh ${ }^{2}$ \\ ${ }^{1}$ Department of Anesthesiology and Pain Medicine, Catholic Kwandong University of Korea College of Medicine, \\ International St. Mary's Hospital, Incheon, Republic of Korea \\ ${ }^{2}$ Department of Anesthesiology and Pain Medicine, Asan Medical Center, University of Ulsan College of Medicine, \\ 88 Olympic-ro 43 Gil, Songpa-gu, Seoul 05505, Republic of Korea
}

Correspondence should be addressed to Jeong Hun Suh; paindrsuh@gmail.com

Received 28 October 2015; Accepted 30 December 2015

Copyright (C) 2016 Young Uk Kim et al. This is an open access article distributed under the Creative Commons Attribution License, which permits unrestricted use, distribution, and reproduction in any medium, provided the original work is properly cited.

Background. The cervical epidural space can be detected by the loss of resistance (LOR) technique which is commonly performed using air. However, this technique using air has been associated with a high false-positive LOR rate during cervical interlaminar epidural steroid injections (CIESIs). Objective. We investigated whether the detection of LOR with contrast medium might reduce the false-positive LOR rate on the first attempt. Methods. We obtained data retrospectively. A total of 79 patients were divided into two groups according to the LOR technique. Groups 1 and 2 patients underwent CIESI with the LOR technique using air or contrast medium. During the procedure, the injection technique (median or paramedian approach), final depth, LOR technique (air or contrast), total number of LOR attempts, and any side effects were recorded. Results. The mean values for the total number of LOR attempts were $1.38 \pm 0.65$ (Group 1) and $1.07 \pm 0.25$ (Group 2). The false-positive rate on the first attempt was $29.4 \%$ and $6.6 \%$ in Groups 1 and 2, respectively $(P=0.012)$. Conclusions. The use of contrast medium for LOR technique is associated with a lower rate of false-positivity compared with the use of air.

\section{Introduction}

Cervical interlaminar epidural steroid injections (CIESIs) are widely used to treat chronic axial neck and radicular pain from spinal stenosis, herniated discs, and acute pain conditions that involve the upper extremities, posterior neck, and head [1-5]. Loss of resistance (LOR) is the most commonly used technique for identifying the epidural space. It relies on sudden LOR to the injection of either air or liquid. To improve the accuracy of needle placement, many clinicians prefer a fluoroscopic-guided LOR technique [4]. However, several studies of CIESI have reported a $30-53 \%$ false-positive rate with air on the first attempt because of high rate of variability and discontinuity in the ligamentum flavum [6], which plays an essential role in the cervical region $[1,4,7,8]$. Although CIESI is considered to be a relatively safe procedure, the high rate of false-positivity for LOR in the cervical region is associated with significant complications [9].
The primary aim of this study was to examine whether the use of air or contrast medium for the LOR technique could diminish the false-positive rate on the first attempt. The falsepositive rate represents a loss prior to actually entering the epidural space in this study. We predicted that LOR detection with contrast medium would increase the accuracy of CIESI because of the high viscosity and surface tension of this medium.

\section{Methods}

The research protocol was approved by our institutional review board and registered at the University of Ulsan, Seoul, Republic of Korea, with the assigned number S2014-20400001. Procedure records for all relevant patients at Asan Medical Center were requested between June 2014 and June 2015. We retrospectively reviewed these patients medical 


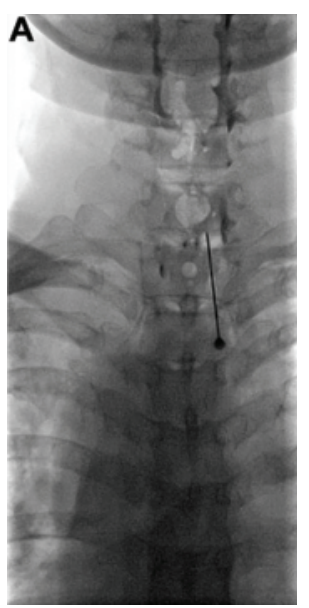

(a)

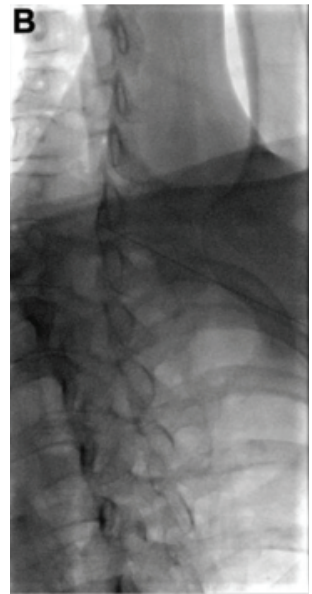

(b)

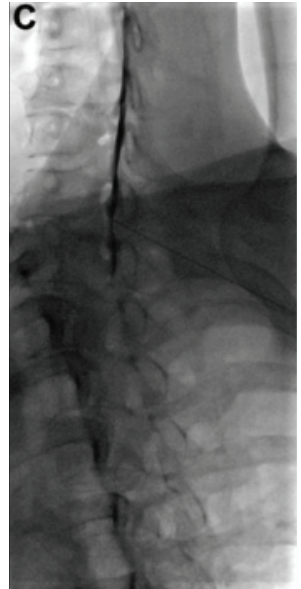

(c)

Figure 1: AP and the contralateral oblique views under fluoroscopy. AP view (a) and the contralateral oblique view at 50 degrees ((b) and (c)) under fluoroscopy; the contralateral laminae are seen in complete cross-section view ((b) and (c)). The needle can be seen transversing between the inferior and superior laminae with the needle tip directed toward the spinolaminar line (b). And epidural space was confirmed with contrast medium injection (c).

records, including demographic data (sex, age, weight, and height) and chief complaints (pain, paresthesia, or both pain and paresthesia with weakness). Short abstracts were written based on these records, including details of the procedure and technique. In our Department of Pain Medicine, cervical spine interventions are recorded using a consistent itemized format. During each procedure, the injection technique (median or paramedian approach), final depth, the LOR technique (air or contrast), total number of LOR procedures, and any side effects, such as a dural puncture, were recorded.

We divided subjects into two groups according to the LOR technique that was used; Group 1 patients underwent CIESI with the LOR technique using air, while Group 2 patients underwent CIESI with the LOR technique using a contrast agent (Omnipaque 300 [iohexol, $300 \mathrm{mg}$ iodine per $\mathrm{mL}$ ]; GE Healthcare, Little Chalfont, UK). Inclusion was limited to patients with a history of chronic functional limitations of the upper extremity, shoulder, and posterior neck because of pain that was of at least 3-month duration and resulted from cervical disc herniation or uncovertebral joint narrowing. In addition, only patients 20 years or older were included. All patients with prior cervical surgery, dislocations, and fractures of the cervical spine were excluded. Fluoroscopically guided CIESI was performed by one pain specialist who had completed more than 1000 ESI procedures at Asan Medical Center in a sterile operating room. All patients were positioned in a prone position with the neck flexed, facedown, with two gel-pads stacked under the chest.

In Group 1, the $22 \mathrm{G}$ Tuohy needle (Hakko; Chikumashi, Nagano-ken, Japan) was connected to a syringe that contained $2.5 \mathrm{~mL}$ air, while in Group 2, the $22 \mathrm{G}$ Tuohy needle was filled with contrast medium $(0.2 \mathrm{~mL}$ contrast $)$ and connected to a syringe that contained $2.5 \mathrm{~mL}$ air. Under fluoroscopic visualization, the interlaminar space between C7 and $\mathrm{T} 1$ was identified. After anesthetizing the skin with $1 \%$ lidocaine, a 22-gauge Tuohy needle was inserted via a midline or paramedian approach that matched the symptomatic side at the C7-T1 interspace (Figure 1(a)).

Cervical contralateral oblique view was used during the procedure. To obtain optimal contralateral oblique view, fluoroscopy was rotated in AP/lateral plane until a parallel view of the contralateral lamina was achieved. When X-ray beam was parallel to the ventral margin of the lamina, we can better visualize the spinolaminar line. The needle was slowly and carefully advanced through the ligamentum flavum using the LOR technique after the level is confirmed. In this fluoroscopic vision, the needle can be seen transversing between the inferior and superior laminae with the needle tip directed toward the spinolaminar line (Figure 1(b)). The posterior epidural space was entered between C7 and T1. Confirmation of correct epidural placement occurred when the contrast agent (Omnipaque 300, GE Healthcare, Little Chalfont, UK) was seen to spread evenly throughout the epidural space (Figure 1(c)). A mixture of $5 \mathrm{mg}$ dexamethasone and 3-6 mL $0.125 \%$ bupivacaine was then injected into the epidural space.

2.1. Statistical Analysis. Data were expressed as means \pm standard deviation (SD). We used an unpaired $t$-test to compare the means of the two study groups. Comparisons of the success rate were made using the Pearson chi-square test and Fisher's exact test. $P$ values $<0.05$ were considered to indicate statistically significant differences. SPSS for Windows version 21 (IBM SPSS Inc., Chicago, IL) was used for statistical analysis.

\section{Results}

A total of 79 patients were included in our current analyses. The demographics of the study subjects managed with LOR 
TABLE 1: Study patient characteristics and the final depth of the cervical epidural space.

\begin{tabular}{lccc}
\hline Parameter & $\begin{array}{c}\text { Air group } \\
(N=34)\end{array}$ & $\begin{array}{c}\text { Contrast group } \\
(N=45)\end{array}$ & $P$ value \\
\hline Age $(\mathrm{yr})$ & $55.65 \pm 12.52$ & $52.58 \pm 13.87$ & 0.441 \\
Height $(\mathrm{cm})$ & $167.85 \pm 8.03$ & $165.68 \pm 7.35$ & 0.361 \\
Weight $(\mathrm{kg})$ & $65.81 \pm 8.70$ & $62.84 \pm 10.01$ & 0.295 \\
BMI $\left(\mathrm{kg} / \mathrm{m}^{2}\right)$ & $21.71 \pm 6.77$ & $22.79 \pm 2.49$ & 0.509 \\
Diagnosis $(N)$ & & & \\
$\quad$ C-HIVD & 22 & 34 & \\
$\quad$ C-SS & 12 & 11 & \\
Needle tip approach & & & \\
$(N)$ & & & \\
$\quad$ Median approach & 0 & 44 & \\
Paramedian & 34 & 24 & \\
approach & 18 & 20 & \\
$\quad$ Right & 16 & $6.69 \pm 0.85$ & 0.062 \\
$\quad$ Left & $6.25 \pm 0.72$ & & \\
Final depth $(\mathrm{cm})$ & & & \\
\hline
\end{tabular}

BMI: body mass index; C-HIVD: cervical herniated intervertebral disc; $\mathrm{C}$ SS: cervical spinal stenosis.

TABLE 2: Total number of LOR procedures and unintentional dural punctures.

\begin{tabular}{lcc}
\hline Variable & $\begin{array}{c}\text { Air group } \\
(N=34)\end{array}$ & $\begin{array}{c}\text { Contrast group } \\
(N=45)\end{array}$ \\
\hline Total number of LOR & 24 & 42 \\
1 & 7 & 3 \\
2 & 3 & 0 \\
3 & 0 & 0 \\
4 or more & $1.38 \pm 0.65$ & $1.07 \pm 0.25$ \\
Mean values & 0 & 0 \\
Unintentional dural puncture & $N)$ &
\end{tabular}

LOR: loss of resistance.

using air or contrast are listed in Table 1. There were no significant differences in any of the patient characteristics between the two study groups (Table 1). The total number of LOR attempts is indicated in Table 2 . The mean values for the total number of LOR attempts were $1.38 \pm 0.65$ (air group) and $1.07 \pm 0.25$ (contrast group). In Group 1, 24 patients exhibited successful results (successful identification of the epidural space by the LOR technique on the first attempt) and 10 patients were classified as a fail (success rate $70.6 \%$ and false-positive rate 29.4\%). In contrast, in Group 2 there were 42 successes and 3 fails (success rate $93.4 \%$ and false-positive rate $6.6 \%$; Table 3 ). This difference was significant $(P=0.012$, Fisher's exact test). The success rate was significantly lower in the air group (Group 1) than in the contrast group (Group 2 ; odds ratio $=0.171 ; 95 \%$ confidence interval $[\mathrm{CI}], 0.043$ to $0.684)$.
TABLE 3: Comparison of the success rate on the first attempt.

\begin{tabular}{lccc}
\hline & \multicolumn{2}{c}{ Group } & Total \\
& Air & Contrast & \\
\hline First attempt & & & \\
Success & 24 & 42 & 66 \\
Failure & 10 & 3 & 13 \\
Total & 34 & 45 & 79 \\
\hline
\end{tabular}

\section{Discussion}

Although CILESIs are commonly used procedures in the diagnosis and treatment of painful disorders of the cervical spine [10], they have been associated with complications, such as nonpositional headache, facial flushing, vasovagal episodes, and increased axial neck pain. Additionally, major complications, such as epidural hematoma, subdural hematoma, permanent spinal cord injury, and death, can occur during the procedure $[11,12]$. To improve patient safety, many clinicians recommend performing the procedure under fluoroscopic guidance [4, 13-15]. Fluoroscopicguided procedures allow the injection of contrast medium to verify the correct spread of the injectate in the cervical epidural space and to exclude intravascular or intrathecal injection [16]. However, fluoroscopic guidance CILESI does not diminish the false-positive rate on the first attempt. No previous prospective or retrospective studies have compared the false-positive rate in the cervical region when air or contrast is used.

Stojanovic et al. reported a $30-53 \%$ false-positive LOR rate during the first attempt to identify the epidural space [4]; these authors performed the LOR technique using air. Our current findings are consistent with that previous report and indicate a false-positive rate of $29.4 \%$ on the first attempt to identify the epidural space by LOR detection using air. We found in comparison that a contrast-filled Tuohy needle for the LOR technique was associated with a lower false-positive rate $(6.6 \%)$. We used an iohexol preparation (Omnipaque 300, GE Healthcare, Little Chalfont, UK), which contains $647 \mathrm{mg}$ iohexol (equivalent to $300 \mathrm{mg}$ organic iodine per $\mathrm{mL}$ ) and is provided as a colorless to pale-yellow, pyrogen-free sterile solution. The osmolarity of iohexol is $465 \mathrm{mOsm} / \mathrm{L}-$ approximately 1.5 -fold greater than that of normal saline. A cadaveric study has demonstrated that liquid injected under pressure at the moment of LOR can potentially thrust the dura away from the needle tip, thereby helping to avoid dural puncture when compared to air [17]. We speculate that contrast is more effective than saline in this regard because of its greater osmolality and viscosity (the Omnipaque 300 viscosity at $37.0^{\circ} \mathrm{C}$ is 6.3 centipoise (cp), whereas the normal saline viscosity at $37.0^{\circ} \mathrm{C}$ is $0.8 \mathrm{cp}$ ).

We did not compare contrast with saline because Segal and Arendt have reported that the isotonic saline returning from a blocked needle might sometimes be mistaken for cerebrospinal fluid when the Tuohy needle is not yet in the epidural space, resulting in a failed block [3]. In the cervical region, anatomical studies have shown not only high rate of discontinuity, but also anatomic variability in the ligamentum 
flavum, which plays an inevitable role in the LOR technique. This morphological variability is associated with a higher false-positive rate during CIESI procedures [7, 18, 19].

According to the epidural steroid injections safety recommendations, entry at C7-T1 level is recommended, but preferably not higher than the C6-C7 level because of the variability and discontinuity of the ligamentum flavum. Therefore, we performed CILESI at C7-T1 level following recommendations [20, 21]. Although fluoroscopic-guided techniques increase the procedure precision and help to confirm correct needle placement [7, 14, 22], the LOR technique with air has a high rate of false-positive LOR on the first attempt.

It is known that performing the LOR technique with air can increase the incidence of complications, such as venous air emboli, nerve root compression, and subcutaneous emphysema $[23,24]$. Epidural air can migrate around the nerve roots. Additionally, depending on its location, neurological complications, such as cervical root compression, multiradicular syndrome, and even paraplegia, can occur [12]. Therefore, LOR with air injection can cause radiculopathy. The potential complications of air also include pneumocephalus and headaches in patients who receive epidural anesthesia. These headaches from pneumocephalus result from an accidental dural puncture during epidural placement using air for the LOR technique [25]. To prevent these complications, the LOR technique using contrast medium should be considered, which could potentially diminish the incidence of certain air-related complications. Furthermore, the cervical epidural space can be detected directly by contrast medium that is distributed using a Tuohy needle without additional contrast agent and could thus help to reduce radiation exposure. Finally, we detected a statistically significant reduction in the number of attempts required to locate the epidural space with LOR using contrast medium instead of air.

We used contralateral oblique view image because the shoulders can obscure visual identification of epidural space in true lateral view image of C7-T1 [26]. Gill et al. have demonstrated that the contralateral oblique view in the cervicothoracic level is superior to the lateral view for the aim of needle tip visualization and in providing a consistent landmark for evaluating the epidural space [27].

Our study had several limitations of note. First, it was not a prospective, controlled study. However, followup records were recorded on an itemized medical chart such that the follow-up data could be more systemically gathered in a manner similar to that used in prospective studies. Second, the sample size was relatively small. Future studies should include a larger number of subjects to further evaluate differences between air-only, saline-only, and contrast-only LOR techniques. The findings of our present pilot study may yield insights into the expected differences in these LOR methods and help to enhance the statistical power of future studies. Third, the falsepositive rate only represents a loss prior to actually entering the posterior epidural space in our study. This falsepositive does not include intrathecal or intramedullary access.

\section{Conclusions}

The use of contrast medium for the LOR technique is associated with a lower false-positive rate on the first attempt compared with air. Additionally, LOR techniques using contrast can shorten the procedure time and limit radiation exposure due to the reduced false-positive rate. We thus strongly recommend this approach when performing CIESI.

\section{Additional Points}

We surveyed total number of loss of resistance attempts in patients who underwent cervical interlaminar epidural steroid injection with the loss of resistance technique using either air (Group 1) or contrast medium (Group 2). The loss of resistance technique using contrast medium showed a lower false-positive rate compared with the use of air. This technique can reduce the procedure time and limit radiation exposure on account of the reduced false-positive rate. In consequence, loss of resistance maneuver using contrast medium should be considered when performing cervical interlaminar epidural steroid injection.

\section{Competing Interests}

The authors declare that they have no competing interests.

\section{Authors' Contributions}

Role. Young Uk Kim and Doohwan Kim equally contributed to this paper as co-first authors. Young Uk Kim, Doohwan Kim, and Jeong Hun Suh helped design the study, conduct the study, analyze the data, and write the paper. Jun Young Park, Jae-Hyung Choi, Ji Hyun Kim, Heon-Yong Bae, and Eun-Young Joo helped conduct the study and analyze the data. Attestation. Young Uk Kim and Jeong Hun Suh have seen the original study data, reviewed the analysis of the data, approved the final paper, and are the authors responsible for archiving the study files. Doohwan Kim, Jun Young Park, JaeHyung Choi, Ji Hyun Kim, Heon-Yong Bae, and Eun-Young Joo have seen the original study data, reviewed the analysis of the data, and approved the final paper.

\section{References}

[1] L. Manchikanti, K. A. Cash, V. Pampati, B. W. Wargo, and Y. Malla, "The effectiveness of fluoroscopic cervical interlaminar epidural injections in managing chronic cervical disc herniation and radiculitis: preliminary results of a randomized, doubleblind, controlled trial," Pain Physician, vol. 13, no. 3, pp. 223236, 2010.

[2] L. Manchikanti, K. A. Cash, V. Pampati, B. W. Wargo, and Y. Malla, "Management of chronic pain of cervical disc herniation and radiculitis with fluoroscopic cervical interlaminar epidural injections," International Journal of Medical Sciences, vol. 9, no. 6, pp. 424-434, 2012.

[3] S. Segal and K. W. Arendt, "A retrospective effectiveness study of loss of resistance to air or saline for identification of the epidural 
space," Anesthesia and Analgesia, vol. 110, no. 2, pp. 558-563, 2010.

[4] M. P. Stojanovic, T.-N. Vu, O. Caneris, J. Slezak, S. P. Cohen, and C. N. Sang, "The role of fluoroscopy in cervical epidural steroid injections: an analysis of contrast dispersal patterns," Spine, vol. 27, no. 5, pp. 509-514, 2002.

[5] L. Manchikanti, K. A. Cash, V. Pampati, and Y. Malla, “Two-year follow-up results of fluoroscopic cervical epidural injections in chronic axial or discogenic neck pain: a randomized, doubleblind, controlled trial," International Journal of Medical Sciences, vol. 11, no. 4, pp. 309-320, 2014.

[6] Q. H. Hogan, "Epidural anatomy examined by cryomicrotome section. Influence of age, vertebral level, and disease," Regional Anesthesia, vol. 21, no. 5, pp. 395-406, 1996.

[7] R. Lieberman, P. Dreyfuss, and R. Baker, "Fluoroscopically guided interlaminar cervical epidural injections," Archives of Physical Medicine and Rehabilitation, vol. 84, pp. 1568-1569, 2003.

[8] A. H. White, "Injection techniques for the diagnosis and treatment of low back pain," Orthopedic Clinics of North America, vol. 14 , no. 3, pp. 553-567, 1983.

[9] K. P. Botwin, R. Castellanos, S. Rao et al., "Complications of fluoroscopically guided interlaminar cervical epidural injections," Archives of Physical Medicine and Rehabilitation, vol. 84, no. 5, pp. 627-633, 2003.

[10] R. C. Huang, G. S. Shapiro, M. Lim, H. S. Sandhu, G. E. Lutz, and R. J. Herzog, "Cervical epidural abscess after epidural steroid injection,” Spine, vol. 29, no. 1, pp. E7-E9, 2004.

[11] R. Maddela, S. E. Wahezi, S. Sparr, and A. Brook, "Hemiparesis and facial sensory loss following cervical epidural steroid injection," Pain Physician, vol. 17, no. 6, pp. E761-E767, 2014.

[12] A. Abbasi, G. Malhotra, G. Malanga, E. P. Elovic, and S. Kahn, "Complications of interlaminar cervical epidural steroid injections: a review of the literature," Spine, vol. 32, no. 19, pp. 2144-2151, 2007.

[13] R. Cluff, A.-K. Mehio, S. P. Cohen, Y. Chang, C. N. Sang, and M. P. Stoianovic, "The technical aspects of epidural steroid injections: a national survey," Anesthesia and Analgesia, vol. 95, no. 2, pp. 403-408, 2002.

[14] K. S. Kim, S. S. Shin, T. S. Kim, C. Y. Jeong, M. H. Yoon, and J. I. Choi, "Fluoroscopically guided cervical interlaminar epidural injections using the midline approach: an analysis of epidurography contrast patterns," Anesthesia \& Analgesia, vol. 108, no. 5, pp. 1658-1661, 2009.

[15] L. Manchikanti, Y. Malla, K. A. Cash, C. D. Mcmanus, and V. Pampati, "Fluoroscopic cervical interlaminar epidural injections in managing chronic pain of cervical postsurgery syndrome: preliminary results of a randomized, double-blind, active control trial," Pain Physician, vol. 15, no. 1, pp. 13-26, 2012.

[16] M. H. Landers, P. Dreyfuss, and N. Bogduk, "On the geometry of fluoroscopy views for cervical interlaminar epidural injections," Pain Medicine, vol. 13, no. 1, pp. 58-65, 2012.

[17] W. P. Mckay, T. Rosser, S. Kriegler, and A. Mohamed, "Epidural loss-of-resistance biomechanics: an open pilot cadaver study," Local and Regional Anesthesia, vol. 3, no. 1, pp. 101-107, 2010.

[18] M. K. Fujinaka, E. F. Lawson, G. Schulteis, and M. S. Wallace, "Cervical epidural depth: correlation between needle angle, cervical anatomy, and body surface area," Pain Medicine, vol. 13, no. 5, pp. 665-669, 2012.

[19] S. P. Yoon, H. J. Kim, and Y. S. Choi, "Anatomic variations of cervical and high thoracic ligamentum flavum," Korean Journal of Pain, vol. 27, no. 4, pp. 321-325, 2014.
[20] P. Lirk, J. Colvin, B. Steger et al., "Incidence of lower thoracic ligamentum flavum midline gaps," British Journal of Anaesthesia, vol. 94, no. 6, pp. 852-855, 2005.

[21] L. Manchikanti, F. J. E. Falco, R. M. Benyamin, C. G. Gharibo, K. D. Candido, and J. A. Hirsch, "Epidural steroid injections safety recommendations by the multi-society pain workgroup (MPW): more regulations without evidence or clarification," Pain Physician, vol. 17, no. 5, pp. E575-E588, 2014.

[22] J. W. Lee, S. Y. Hwang, G. Y. Lee, E. Lee, and H. S. Kang, "Fluoroscopic cervical paramidline interlaminar epidural steroid injections for cervical radiculopathy: effectiveness and outcome predictors," Skeletal Radiology, vol. 43, no. 7, pp. 933-938, 2014.

[23] M. H. Lee, C. S. Han, S. H. Lee et al., "Motor weakness after caudal epidural injection using the air-acceptance test," Korean Journal of Pain, vol. 26, no. 3, pp. 286-290, 2013.

[24] H. K. Lim, Y. D. Cha, J. H. Song, J. W. Park, and M. H. Lee, "Asymptomatic pneumomediastinum resulting from air in the epidural space -a case report," Korean Journal of Anesthesiology, vol. 65, no. 3, pp. 266-269, 2013.

[25] C. L. Sanford II, R. E. Rodriguez, J. Schmidt, and P. N. Austin, "Evidence for using air or fluid when identifying the epidural space," AANA Journal, vol. 81, no. 1, pp. 23-28, 2013.

[26] M. Furman, N. R. Jasper, and H. W. Lin, "Fluoroscopic contralateral oblique view in interlaminar interventions," Pain Medicine, vol. 13, no. 11, pp. 1389-1396, 2012.

[27] J. S. Gill, M. Aner, N. Jyotsna, J. C. Keel, and T. T. Simopoulos, "Contralateral oblique view is superior to lateral view for interlaminar cervical and cervicothoracic epidural access," Pain Medicine, vol. 16, no. 1, pp. 68-80, 2015. 


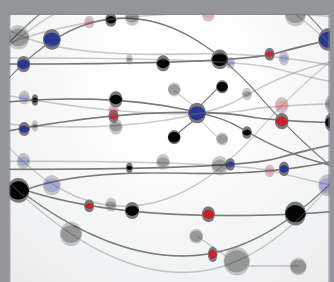

The Scientific World Journal
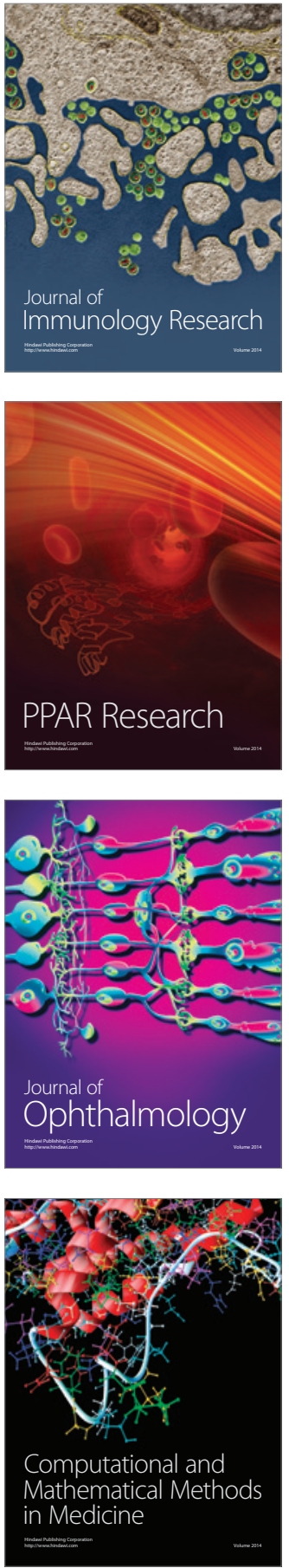

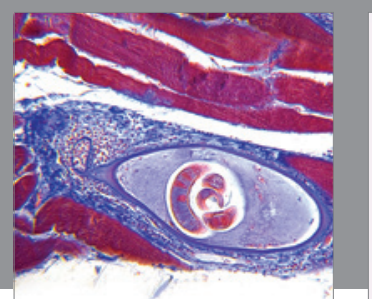

Gastroenterology Research and Practice

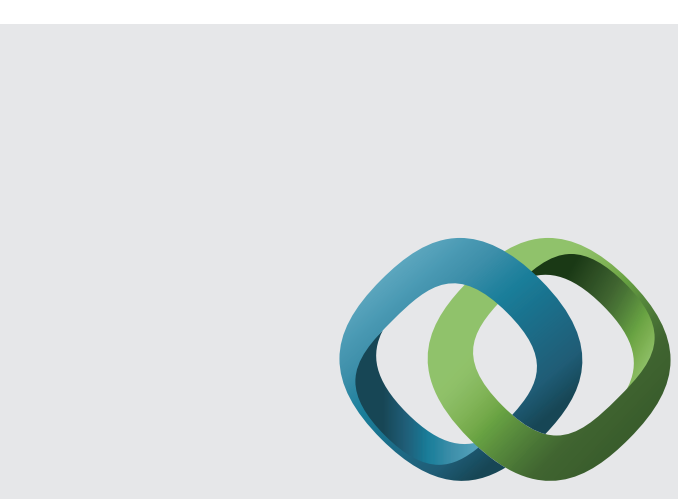

\section{Hindawi}

Submit your manuscripts at

http://www.hindawi.com
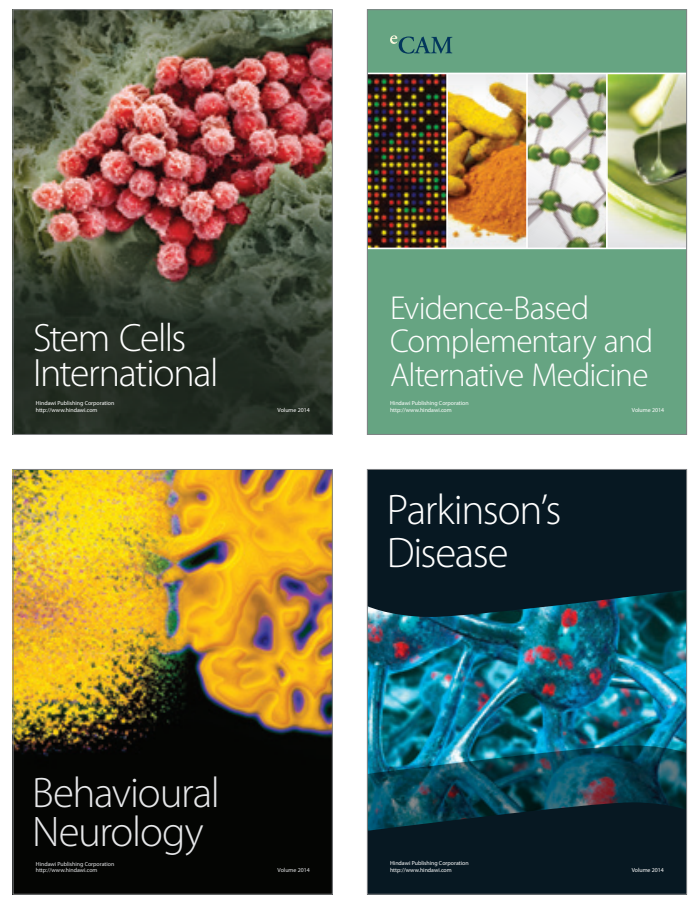
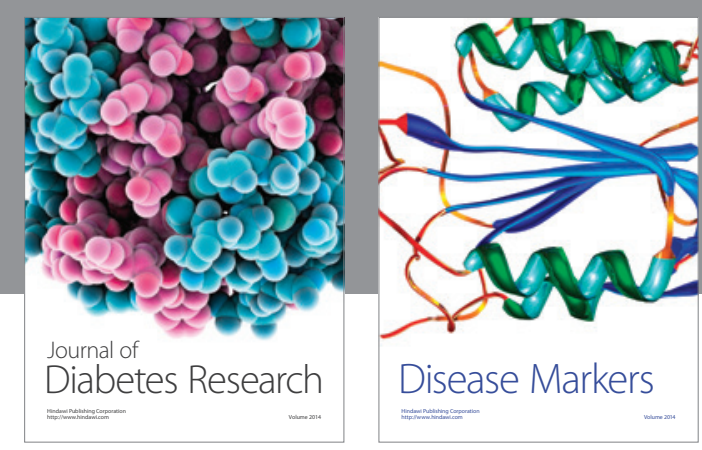

Disease Markers
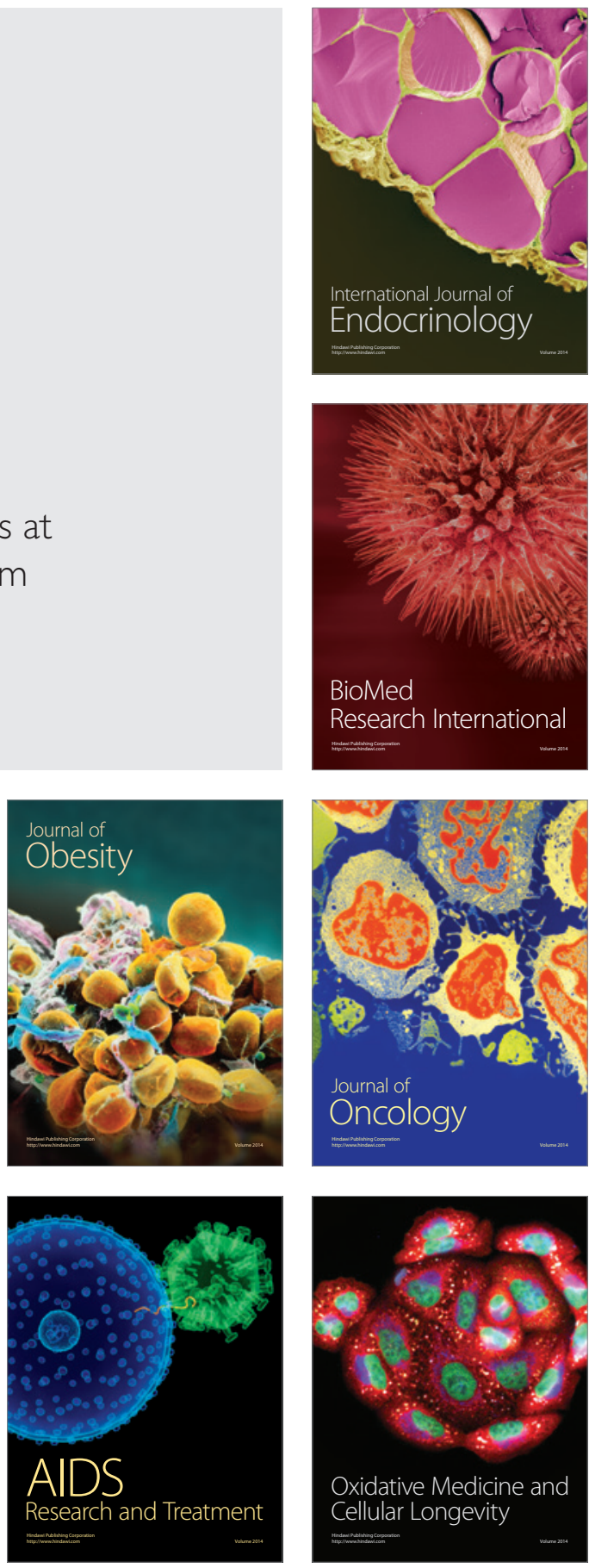